\title{
The management of fever and petechiae: making sense of rash decisions
}

\author{
P A Brogan, A Raffles
}

\begin{abstract}
In a retrospective and prospective audit of 55 children presenting to the paediatric assessment unit of a district general hospital with fever and petechial rash, 9\% had significant bacterial sepsis. The "ILL criteria" (irritability, lethargy, low capillary refill) for the management of children with fever and petechiae are proposed. (Arch Dis Child 2000;83:506-507)
\end{abstract}

Keywords: fever; petechial rash; bacterial sepsis; risk factor; meningococcaemia

Not all children with fever and petechiae have invasive bacteraemia - in fact the minority do. ${ }^{1-3}$ Currently there are no guidelines concerning the management of this patient group, and the tendency is for these children to default into treatment regimens for meningococcaemia.

The aims of this study were to identify risk factors predictive of significant bacterial sepsis (SBS) in children with fever and petechiae, and to establish a set of clinical guidelines to aid the management of this patient group.

Department of Paediatrics, QEII

Hospital, Howlands, Welwyn Garden City,

Herts, UK

P A Brogan

A Raffles

Correspondence to: Dr P A Brogan, Department of Nephrourology, Institute of Child Health, Level 2, 30 Guilford St, London WC1N 1EH, UK

Pbrogan@ich.ucl.ac.uk

Accepted 17 July 2000

Table 1 Clinical and laboratory features of patients identified with significant bacteraemia ing, or medical staff); abnormality of the peripheral blood white cell count (WCC) ${ }^{45}$ (total WCC outside the range 5-15 $\times 10^{9} / 1$ ); elevation of $\mathrm{C}$ reactive protein ${ }^{6}$ (CRP greater than $5 \mathrm{mg} / \mathrm{l}$ ).

A "well" patient was defined as a patient who did not manifest any of the proposed risk factors for SBS. An "unwell" patient was defined as a patient manifesting one or more risk factors for SBS. Culture negative sepsis was defined as patients who appeared clinically toxic, but in whom no organism was isolated.

\section{Results}

Fifty five patients (median age 2.52 years, range $0.22-15.82$ ) satisfying entry criteria presented during the audit periods (November 1997 through April 1998; July 1998 through January 1999). Five of these patients (9\%) had SBS (table 1). Figure 1 shows overall diagnoses.

The performance of the combined risk factors as a screening test for the prediction of SBS based only on those patients who had blood cultures performed ( $\mathrm{n}=33$ ) were as follows: sensitivity $100 \%$ (95\% confidence interval (CI), 48-100\%); specificity 57\% (37-76\%); positive predictive value $29 \%(14-45 \%)$; negative predictive value $100 \%$ (79-100\%); pretest number needed to treat (NNT) 6.7; postpositive test NNT 3.3 .

The results based on all patients $(n=55)$ assuming that those patients who did not have blood cultures performed did not have SBS (no patient died and no patient returned to hospital) were: sensitivity $100 \% \quad(95 \%$ CI, $48-$ $100 \%)$; specificity $60 \%(45-74 \%)$; positive predictive value $20 \%$ (91-31\%); negative predictive value $100 \%(88-100 \%)$; pretest NNT 11.1; post-positive test NNT 5.

\begin{tabular}{|c|c|c|c|c|c|c|c|c|c|}
\hline Age (y) & $\operatorname{Sex}$ & $\begin{array}{l}\text { Month of } \\
\text { presentation }\end{array}$ & Clinical features & Rash & Temp. & $\begin{array}{l}W C C \\
\left(\times 10^{9} / l\right)\end{array}$ & $\begin{array}{l}C R P \\
(m g / l)\end{array}$ & Organism isolated & Method of detection \\
\hline 13.4 & $\mathrm{~F}$ & February & Toxic and shocked & $\begin{array}{l}\text { Purpuric (initially } \\
\text { petechial) }\end{array}$ & $38^{\circ} \mathrm{C}$ & 5.3 & 79 & N. meningiditis & + blood culture; + rapid Ag \\
\hline 12.8 & M & February & $\begin{array}{l}\text { Toxic and meningism, } \\
\text { received IM BP }\end{array}$ & Petechial & $40^{\circ} \mathrm{C}$ & 24.5 & 302 & Group B streptococcus & $\begin{array}{l}\text { + rapid } \mathrm{Ag} ; \text { - blood culture } \\
\text { (post IM BP) }\end{array}$ \\
\hline 1.46 & M & August & Not toxic & Petechial & $40.4^{\circ} \mathrm{C}$ & 22.7 & 50 & S. pneumoniae & + blood culture \\
\hline 12.9 & $M$ & January & Toxic & Petechial & $38.9^{\circ} \mathrm{C}$ & 16.8 & 277 & N. meningiditis type $\mathrm{C}$ & + PCR; + blood culture \\
\hline 1.52 & $\mathrm{~F}$ & January & Toxic & $\begin{array}{l}\text { Purpuric (initially } \\
\text { petechial) }\end{array}$ & $40.4^{\circ} \mathrm{C}$ & 15.2 & 45 & N. meningiditis type $\mathrm{B}$ & + PCR; - blood culture \\
\hline
\end{tabular}

IM BP, intramuscular benzylpenicillin; PCR, polymerase chain reaction; +, positive; -, negative; Temp., temperature; Ag, antigen; WCC, white cell count; CRP, c-reactive protein. 


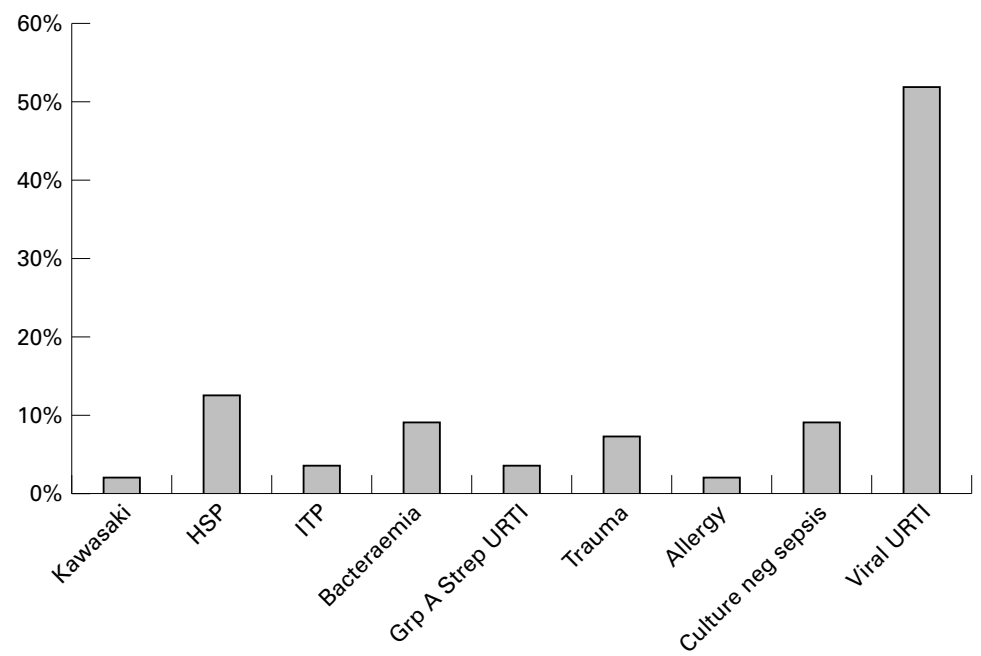

Figure 1 Overall diagnoses $(n=55)$. HSP, Henoch-Schonlein purpura; ITP, idiopathic thrombocytopenic purpura; URTI, upper respiratory tract infection.

\section{Discussion}

The combined risk factors performed as a highly sensitive screening test for SBS in this patient group. In terms of specificity, the combined risk factors performed less well. Nonetheless, only five children would receive unnecessary treatment for every one child treated for invasive bacteraemia if the proposed risk criteria were used to select those patients for treatment.

Not all units have access to out of hours/ weekend CRP results. All of the patients with SBS in our series would have been identified using the combined risk factors excluding $\mathrm{CRP}$, as the presence of any one risk factor defined a child as "unwell", and all the septic patients had at least one risk factor other than elevation of CRP. No attempt has been made to assess the screening test characteristics of any single risk factor taken in isolation - this would require further validation in a larger cohort of patients. Nonetheless, exclusion of the CRP result could conceivably result in some loss of sensitivity of the screening test as a whole, as this risk factor was the only one present in all our patients with SBS.

A previous study examining variation in serum CRP in 124 cases of paediatric meningococcal disease showed lower CRP concentrations at presentation in meningococcal septicaemia than meningococcal meningitis. ${ }^{6}$ Although the median CRP at initial presentation in those with meningococcal septicaemia was $42.6 \mathrm{mg} / \mathrm{l}$, eight children, five of whom had symptoms of less than 12 hours duration, had CRP concentrations on admission that were less than $20 \mathrm{mg} / 1$. These data suggest that over reliance on serum CRP in isolation for the detection of early meningococcaemia should not be recommended.

Similar caution should be exercised regarding the WCC taken in isolation as a means of predicting meningococcal sepsis. Kuppermann et al suggested that the full blood count is not routinely helpful for the diagnosis of meningococcal disease in febrile children, although only one patient of 45 in this retrospective series with "unsuspected meningococcal disease" had petechial rash at presentation. ${ }^{7}$ Pollard et al also suggested that reliance on the WCC as an indicator of meningococcal disease is dangerous, based on the finding that of 128 children with meningococcal sepsis admitted to a single unit, $71 \%$ had a count less than $15 \times 10^{9} / 1 .^{8}$

One important anxiety is the rapidly fulminating course with which invasive meningococcaemia can present. A possible way to overcome this problem is to incorporate a period of observation into any algorithm for patient management, such that any well child with fever and petechiae would be observed for a minimum of four hours and then reassessed prior to discharge.

In conclusion, we propose a new clinical guideline for the management of children presenting with fever and petechiae. We have named the risk factors for SBS the "ILL" criteria as an aide-mémoire: irritabilty, lethargy, low capillary refill and also WCC outside the range $5-15 \times 10^{9} / 1$, and CRP greater than $5 \mathrm{mg} / \mathrm{l}$. We propose that children with fever and petechial rash who have none of the "ILL" criteria and normal WCC and CRP could be observed for a minimum of four hours and reassessed. If no deterioration occurred then outpatient or ambulatory care would be recommended. Children who were not "ILL" but had abnormality of the WCC and/or CRP could be treated for meningococcal disease with a minimum of 48 hours antibiotics pending blood cultures, with notification of "suspected" meningococcal disease, and consideration of rifampicin prophylaxis to family members. Lastly, those children who were "ILL" as defined by the presence of one or more of the proposed criteria, and who had abnormality of the WCC and/or CRP would be treated as meningococcal sepsis, with consideration of early intensive care referral.

We suggest that more studies will be required to validate this guideline; however, we believe that it could prove helpful in the management of this patient group.

1 Mandl KD, Stack AM, Fleisher GR. Incidence of bacteraemia in infants and children with fever and petechiae. $\mathcal{F}$ Pediatr 1997;131:389-404.

2 Baker RC, Seguin JH, Leslie N, Gilchrist MJR, Myers G. Fever and petechiae in children. Pediatrics 1989;84:1051-5.

3 Nguyen QV, Nguyen EA, Weiner LB. Incidence of invasive bacterial disease in children with fever and petechiae. Pediatrics 1984;74:77-80.

4 Dagan R, Powell KR, Hall CB, Menegus MA. Identification of infants unlikely to have serious bacterial infection although hospitalised for suspected sepsis. $\mathcal{F}$ Pediatr 1985;107:855-60.

5 McCarthy CA, Powell KR, Jaskiewicz JA, et al. Outpatient management of selected infants younger than 2 months of age evaluated for possible sepsis. Pediatr Infect Dis f 1990;9: age evalua

6 Marzouk O, Bestwick K, Thomson AP, Sills JA Hart CA. Variation in serum C-reactive protein across the clinical spectrum of meningococcal disease. Acta Paediatr 1993;82: $729-33$.

7 Kuppermann N, Malley R, Inkelis SH, Fleisher GR. Clinical and hematologic features do not reliably identify children with unsuspected meningococcal disease. Pediatrics 1999;103:E20.

8 Pollard AJ, DeMunter C, Nadel S, Levin M. Abandoning empirical antibiotics for febrile children. Lancet 1997;350: 811-12. 